
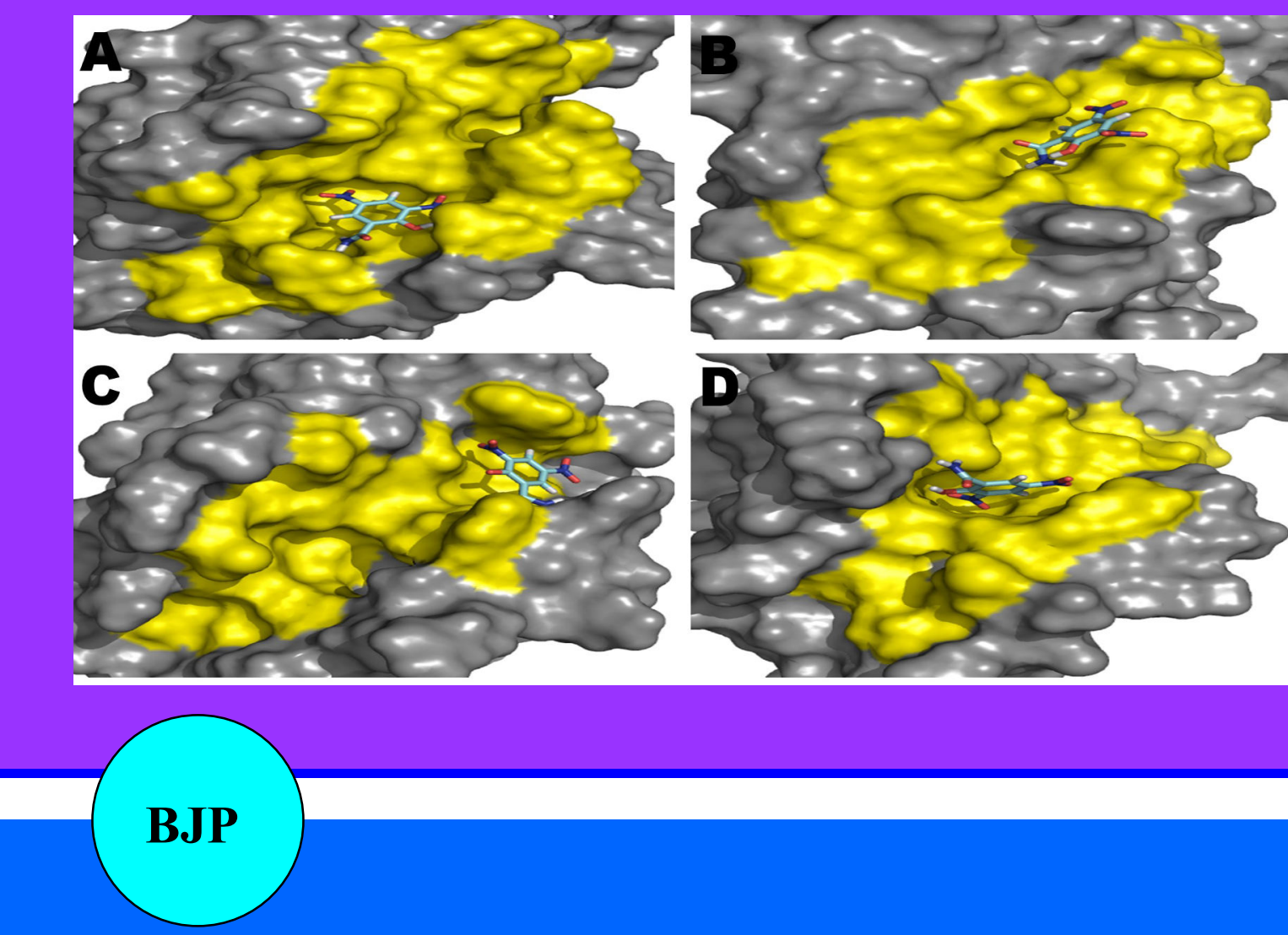

Bangladesh Journal of Pharmacology

Research Article

Computational analyses on delineating specificity of 2-hydroxy-3,5dinitrobenzamide, a BH3-mimetic, towards anti-apoptotic proteins 


\title{
Computational analyses on delineating specificity of 2-hydroxy-3,5 -dinitrobenzamide, a BH3-mimetic, towards anti-apoptotic proteins
}

\author{
Dakshinamurthy Sivakumar'1, Natarajan Hari ${ }^{2}$ and Thirunavukkarasu Sivaraman' \\ ${ }^{1}$ Structural Biology Lab, Department of Bioinformatics, School of Chemical and Biotechnology, SASTRA University, \\ Thanjavur 613 401, Tamil Nadu, India; '2Department of Biotechnology, School of Chemical and Biotechnology, \\ SASTRA University, Thanjavur 613 401, Tamil Nadu, India.
}

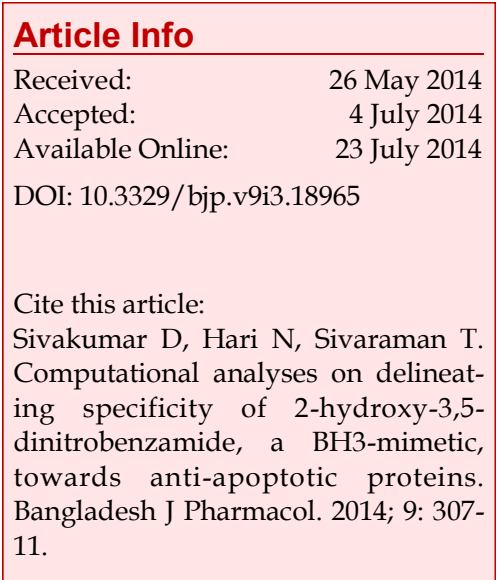

\begin{abstract}
We have herein computationally examined binding affinities and specificity of 2-hydroxy-3,5-dinitrobenzamide (HDNB), a small chemical molecular BH3mimetic identified by means of 'peptidodynmimetic method', on the BH3binding grooves of six anti-apoptotic proteins (Bcl-2, Bcl-B, Bcl-W, Bcl-XL, Bfl1 and Mcl-1) from human beings. The HDNB ligand was found to interact on the $\mathrm{BH} 3$-binding grooves of Bcl-2, Bcl-B, Bcl-XL, Bfl-1 and $\mathrm{Mcl}-1$, whereas it did not act as BH3-mimetic to Bcl-W. Moreover, binding affinities of the HDNB towards the anti-apoptotic proteins were significantly different from each other. The differential binding affinities and specificity of the HDNB towards the anti-apoptotic proteins have been chiefly attributed to the differences in the chemical properties of $\mathrm{BH} 3$-binding grooves of the proteins. Implications of the study to design efficient de novo antagonists to the antiapoptotic proteins using the HDNB as seed molecule have been discussed.
\end{abstract}

\section{Introduction}

Apoptosis is tightly regulated by intrinsic pathway activated by Bcl-2 family proteins present in the mitochondrial membrane and anti-apoptotic proteins of the Bcl-2 family are important cancer biomarkers as they are over expressed in all types of cancers (Kelly and Strasser, 2011). The anti-apoptotic proteins are also playing essential roles in various biological activities: the $\mathrm{Bcl}-2$ and the $\mathrm{Bcl}-\mathrm{XL}$ are playing essential roles for regulating B-lymphocytes and platelet survival, respectively; similarly, Mcl-1 and Bcl-W are essential for the sustainable production of hematopoetic stem cells and sperm cells, respectively (Lessene et al., 2008). Therefore, administration of anti-cancer drugs interacting with anti-apoptotic proteins exhibiting no over expression may presumably cause some adverse effect (such as lymphopenia, thrombocytopaenia and anemia) to cancer patients. In these contexts, examining binding affinities and specificities of antagonists to the anti-apoptotic proteins is crucial to avoid/reduce latestage attritions in drug discovery processes.

We have recently reported 2-hydroxy-3,5-dinitrobenzamide (HDNB) as a drug-likeness inhibitor to human $\mathrm{Bcl}$ -B (hBcl-B) by using an array of computational strategies (Sivakumar et al., 2013). However, binding preferences and specificity of the HDNB towards other five anti-apoptotic proteins have been left unaddressed to date.

In the present study, we have studied binding affinities of the HDNB with each anti-apoptotic protein using molecular docking methods and also stringently scrutinized mode of interactions of the lead with the proteins. Moreover, implications of the study to design highly potent de novo inhibitors to the anti-apoptotic proteins have also been discussed. 


\section{Materials and Methods}

Selections and preparations of anti-apoptotic proteins: Experimental three-dimensional (3D) structures of all the six anti-apoptotic proteins Bcl-2 (1GJH), Bcl-B (4B4S), Bcl-W (1ZY3), Bcl-XL (2YXJ), Bfl-1 (3MQP) and Mcl-1 (2KBW) were retrieved from protein data bank (http://www.pdb.org). Missing hydrogen atoms were added and bond orders were assigned to all proteins considered, herein, by using Schrodinger suite 9.2 and hydrogen bonds were also defined to the structures based on sample water orientation. Using PROPKA module of the Schrodinger suite 9.2, the protein structures were optimized to $\mathrm{pH}$ 7.0. The resultant structures were energy minimized using 'Steepest descent' algorithm with a tolerance of $1000 \mathrm{KJ} / \mathrm{mol} / \mathrm{nm}$ and step size of 0.01 with required number of minimization steps. The energy minimized structures were subjected to molecular dynamics (MD) simulations in near physiological conditions ( $\mathrm{pH} 7.0,1$ atmospheric pressure and ionic strength of $0.1 \mathrm{M} \mathrm{NaCl}$ ) for $5 \mathrm{~ns}$ at $310 \mathrm{~K}$ by Gromacs 4.5 .1 (prior to productive runs, each protein was equilibrated for 200 ps and 500 ps under NVT and NPT conditions, respectively). In each case, trajectory structures were stored at every 25 ps and the data were analyzed in terms of change in potential energy and RMSD of backbone atoms of the proteins. The data analyses revealed that all proteins could attain equilibrium under the defined conditions well within the time span of $5 \mathrm{~ns}$ MD. A representative structure from the equilibrium phase of each anti-apoptotic protein was selected and used as 3D structures of the proteins for all computational studies performed in the present studies unless otherwise mentioned.

Molecular dockings and analyses of non-covalent interactions in the interfaces of anti-apoptotic proteins - HDNB complexes: 3D structure of 2-hydroxy-3,5dinitrobenzamide (ID: MMs01085173) were downloaded from MMsINC database (http://147.162.61.130/ MMsINC/search/). Using the OPLS2005 force field, all possible ionization states of the compound were processed at $\mathrm{pH} 7.0$ using Ionizer algorithm in the ligand preparation module of Schrodinger suite. The program was allowed to generate all combinations of stereoisomers and all $\mathrm{pH}$-dependent chemical forms of the HDNB. The program was allowed to generate low energy ring conformation (with limitation of one per ligand) of the compound. Grid boxes (having dimensions of $80 \times 80 \times 80 \AA$ ) were set to fully cover the $\mathrm{BH} 3$ binding grooves (composed of helices 2-5) of the anti-apoptotic proteins. All generated structures of the HDNB were docked on the $\mathrm{BH} 3$-binding grooves of the 6 anti-apoptotic proteins by Glide-XP with default parameters except that total numbers of poses per run, total numbers of generated poses per ligand for molecular docking and selected poses per ligand in the post-docking processes were set as 10,000, 100 and 10, respectively. Scaling factor and partial charge cut-off were set to 0.8 and 0.15 , respectively, for all molecular docking runs of the present study. Various noncovalent interactions for each of the anti-apoptotic HDNB complexes were analyzed using Schrodinger suite 9.2 and PyMol 1.5.0.1 molecular visualization tools.

\section{Results and Discussion}

There are 6 anti-apoptotic proteins from human being as reported in the literature to date. They are named as Bcl-2, Bcl-B, Bcl-W, Bcl-XL, Bfl-1 and Mcl-1. All the antiapoptotic proteins are single polypeptide chains composed of about 250 standard amino acids and they are simple helical proteins (Ke et al., 2001). The antiapoptotic proteins belonging to $\mathrm{Bcl}-2$ family have similar 3D structures depicting well-defined BH3binding groove (Liang and Fesik, 1997). All the antiapoptotic proteins are playing essential roles on regulating/resisting apoptosis. Moreover, each of the proteins plays unique roles in various biological processes, notwithstanding similar 3D structures of the proteins (Placzek et al., 2010). However, exact structureactivity relationships of the proteins have not yet well addressed to our best knowledge. As these antiapoptotic proteins are retarding apoptosis upon inactivating pro-apoptotic proteins and are also over expressed in cancer diseases, designing of small molecular inhibitors to the proteins has become an important strategy in cancer chemotherapy (Labi et al., 2008). Many drug-likeness chemical inhibitors to the anti-apoptotic proteins have been reported by means of experimental/computational methods in the literature (Sivakumar et al., 2012). In these contexts, we have recently reported 2-hydroxy-3,5-dinitrobenzamide (HDNB) as a novel BH3-mimetic to hBcl-B using peptidodynmimetic method (Sivakumar et al., 2013). In the present study, specificity of the HDNB towards the anti -apoptotic proteins has been demonstrated in detail.

Two-dimensional representation of HDNB structure is shown in Figure 1. The molecule has a six-member ring substituted by four charged chemical moieties. The ligand was docked on the $\mathrm{BH} 3$-binding grooves of the anti-apoptotic proteins and the docking studies suggested that the HDNB was capable to interact with all the anti-apoptotic proteins. However, the HDNB did not interact on the $\mathrm{BH} 3$-binding groove of $\mathrm{Bcl}-\mathrm{W}$, but did with all other five proteins. GlideScores calculated for the HDNB to interact on the $\mathrm{BH} 3$-binding groove of Bcl-2, Bcl-B, Bcl-XL, Bfl-1 and Mcl-1 were ranged from 3.1 to 4.1 implying that the HDNB has moderate differences in its binding preferences towards the proteins (Table I). The HDNB showed relatively stronger interactions with $\mathrm{Bcl}-\mathrm{XL}$ and $\mathrm{Mcl}-1$ comparing its interactions with $\mathrm{Bcl}-\mathrm{B}, \mathrm{Bcl}-2$ and $\mathrm{Bfl}-1$. The differential binding affinities of the HDNB is intriguing as the $\mathrm{BH} 3$ binding groove of each anti-apoptotic protein is unanimously constituted by residues from helices $2,3,4$ and 5 of the respective proteins (Bernardo et al., 2008; 
Table I

Docking parameters and residues of various non-covalent interactions for anti-apoptotic proteins - HDNB complexes

\begin{tabular}{|c|c|c|c|c|c|}
\hline $\begin{array}{l}\text { Proteins and } \mathrm{Pdb} \\
\text { ID }\end{array}$ & $\begin{array}{l}\text { Glide } \\
\text { score }\end{array}$ & $\mathrm{LEb}$ & $\begin{array}{l}\text { Residues within } 4 \AA \text { to the } \\
\text { ligand }\end{array}$ & Hydrogen bonds* & Salt bridge ${ }^{a}$ \\
\hline Bcl-2 (1GJH) & 3.2 & 0.20 & $\begin{array}{l}\text { F104,Y108, D111, F112, } \\
\text { M115,Q118, V133, L137, A149, } \\
\text { F153 }\end{array}$ & $\begin{array}{l}\text { OE1(Q118)...H3(HDNB) } \\
\text { OD1(D111)....H5(HDNB) } \\
\text { OD2(D111)....H5(HDNB) }\end{array}$ & $\begin{array}{l}\text { OD1(D111)....N2 } \\
\text { (HDNB) }\end{array}$ \\
\hline Bcl-B (4B4S) & 3.3 & 0.20 & $\begin{array}{l}\text { L46, H50, F54, M71, V88, T89, } \\
\text { T92, F93 }\end{array}$ & ND1(H50)....H4(HDNB) & - \\
\hline Bcl-W (1ZY3) & 2.9 & 0.18 & Q80, V81, D83, E84, Q87, T89 & $\begin{array}{l}\mathrm{O}(\mathrm{Q} 80) \ldots . \mathrm{H} 4(\mathrm{HDNB}) \\
\mathrm{OE} 2(\mathrm{E} 84) \ldots . . \mathrm{H} 5(\mathrm{HDNB})\end{array}$ & $\begin{array}{l}\text { OE2(E84)....N2 } \\
(\mathrm{HDNB})\end{array}$ \\
\hline Bcl-XL (2YXJ) & 4.1 & 0.25 & $\begin{array}{l}\text { A93, E96, F97, R100, } \\
\text { Y101,W137, G138, V141, F191, } \\
\text { Y195 }\end{array}$ & - & - \\
\hline Bfl-1 (3MQP) & 3.1 & 0.20 & $\begin{array}{l}\text { V48, N51, L52, V74, M75, K77, } \\
\text { E78, T91, F95 }\end{array}$ & $\begin{array}{l}\mathrm{O}(\mathrm{K} 77) \ldots . . \mathrm{H} 3(\mathrm{HDNB}) \\
\mathrm{OE} 1(\mathrm{E} 78) \ldots . . \mathrm{H} 4(\mathrm{HDNB})\end{array}$ & - \\
\hline Mcl-1 (2KBW) & 3.8 & 0.24 & $\begin{array}{l}\text { H224, A227, F228, M231, V249, } \\
\text { H252, V253, S255, T266, L267, } \\
\text { V270 }\end{array}$ & $\mathrm{O}(\mathrm{H} 252) \ldots . . . \mathrm{H} 4(\mathrm{HDNB})$ & - \\
\hline
\end{tabular}

Bernardo et al., 2010; Bernardo et al., 2011; Schreiber and Keating, 2011). However, the BH3-binding grooves of Bcl-W and Mcl-1 are significantly wider comparing the counterpart regions in Bcl-2, Bcl-B, Bcl-XL and Bfl-1. Moreover, binding locations and orientations of the HDNB on the BH3-binding grooves of the 5 antiapoptotic proteins were also different from each other (Figure 1).

Amide protons of the HDNB were invariably in close proximity to any of the residues such as aspartic acid, glutamic acid, glutamine, histidine and lysine located in the $\mathrm{BH} 3-$ binding grooves of the anti-apoptotic proteins: amide protons in the HDNB could be observed to have hydrogen bond interaction with Gln118, His50, Lys77 and His252 of Bcl-2, Bcl-B, Bfl-1 and Mcl-1, respectively. In the case of $\mathrm{Bcl}-\mathrm{XL}$, amide protons of the HDNB were found to be about $3.1 \AA$ and $4.0 \AA$ away from backbone carbonyl group and side-chain carbonyl group of Glu96 of the protein, respectively. These interactions were not classified as hydrogen bonds (H-bonds) as the distance cutoff used in the Schrodinger suite 9.2 for the present study was $2.5 \AA$ for defining inter molecular H-bonds. Similarly, hydroxyl group of the HDNB was found to be in close contacts with the residues such as Asp111, Thr89, Tyr101, Thr91 and His224 located in the BH3binding grooves of Bcl-2, Bcl-B, Bcl-XL, Bfl-1 and Mcl-1, respectively. While the amide and hydroxyl groups of the HDNB were showed electrostatic interactions with charged and polar amino acids, rest of the ligand portion was surrounded by bulky hydrophobic amino acids such as leucine, methionine, phenylalanine and valine present in the $\mathrm{BH} 3$-binding grooves of the antiapoptotic proteins (Table I). It should be mentioned that the HDNB was unable to bind on the BH3-binding groove of Bcl-W, which was constituted by residues such as Ala48, Phe52 (from helix 2) Leu63 (from helix 3), Arg77, Phe78, Val81, Ser82 (from helix 4) and Trp92, Val96, Ala97, Ala100, Phe101, Tyr150 (from helix 5). Strikingly, the $\mathrm{BH} 3$ - binding groove of the $\mathrm{Bcl}-\mathrm{W}$ was remarkably different from the counterpart regions of other 5 anti-apoptotic proteins: The BH3-binding grooves of the 5 proteins are composed of about $60 \%$ hydrophobic amino acids and rest of regions in the grooves of the proteins was found to be occupied with charged and polar amino acids. In contrary, the groove of Bcl-W was predominantly (about $90 \%$ ) constituted by hydrophobic amino acids. The surface groove of the Bcl -W was found to have only an arginine and a serine residue at positions of 77 and 82, respectively (there were no acidic amino acids). Thus, the differential binding affinities of the HDNB towards Bcl-W and other anti-apoptotic proteins could be presumably rationalized to differences in chemical properties of the $\mathrm{BH} 3$-binding grooves of the respective proteins. On the other hand, the HDNB showed relatively weak interaction (vis-à-vis its interaction with other proteins) on a surface groove composed of residues such as Gln80, Val81, Asp83, Glu84, Gln87 and Thr89 of the hBcl-W (Table I and Figure 1) implying that the HDNB was not acting as BH3-mimetic to the anti-apoptotic protein.

Though the six anti-apoptotic proteins are homologous and have similar three-dimensional structures, over expression patterns of the proteins in cancers are not identical (Yip and Reed, 2008). Moreover, structural stabilities and biological activities of the proteins are 


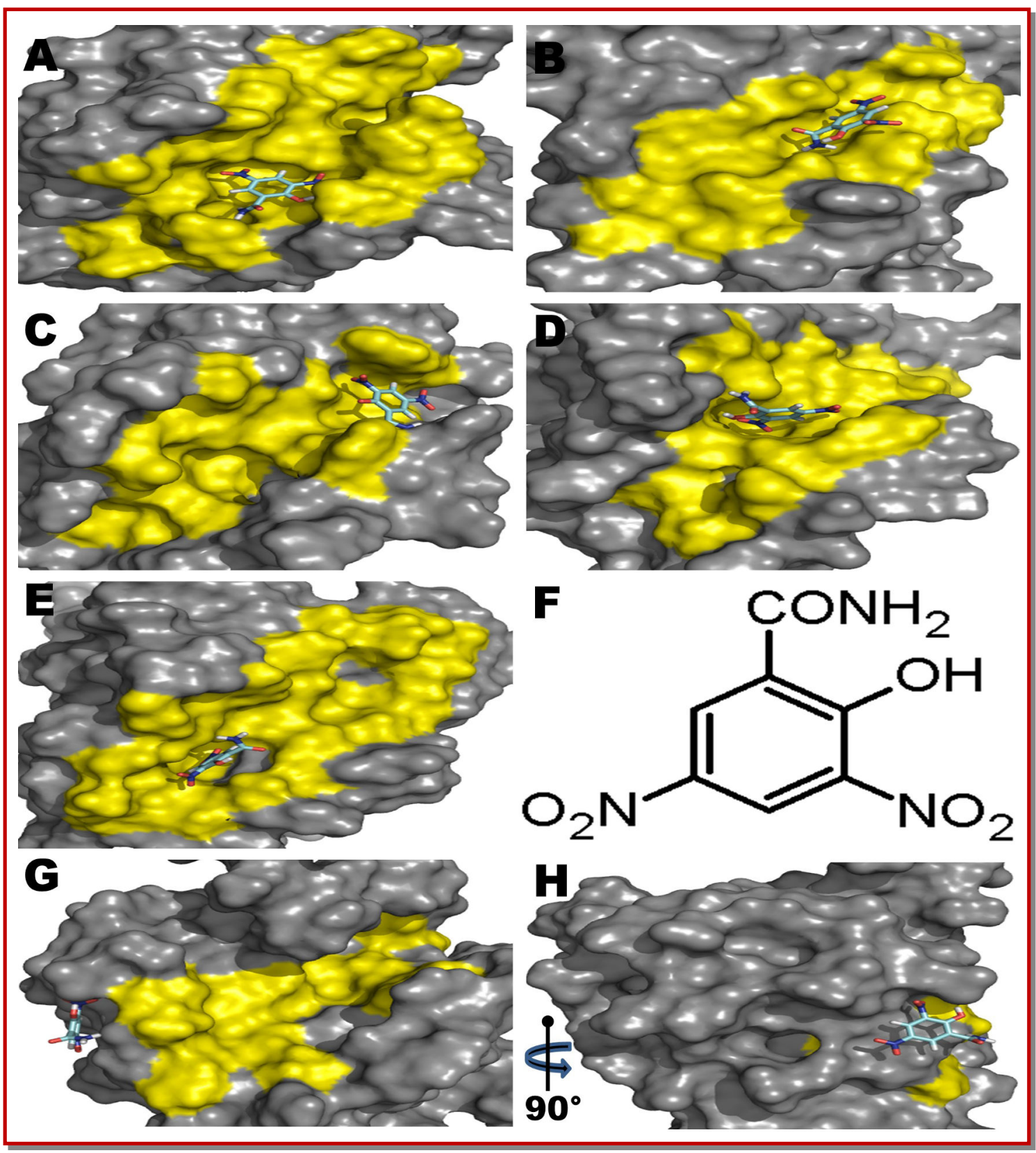

Figure 1: Docking complexes of HDNB and anti-apoptotic proteins $[\mathrm{A}=\mathrm{Bcl}-2, \mathrm{~B}=\mathrm{Bcl}-\mathrm{B}, \mathrm{C}=\mathrm{Bcl}-\mathrm{XL}, \mathrm{D}=\mathrm{Bfl}-1, \mathrm{E}=\mathrm{Mcl}-1, \mathrm{G}=\mathrm{Bcl}-$ $\mathrm{W}$ (front view of $\mathrm{BH} 3$-binding groove) and $\mathrm{H}=\mathrm{Bcl}-\mathrm{W}$ (rear view of $\mathrm{BH}$-binding groove)]. The anti-apoptotic proteins (in similar orientations) and HDNB are shown in surface and sticks models, respectively. The BH3-binding grooves of the anti-apoptotic proteins are painted in yellow color for sake of clarity. F = Two dimensional structure of HDNB (2-hydroxy-3,5-dinitrobenzamide) drawn by using ChemSketch is shown

also different from each other (Reed, 2008). As demonstrated herein and reported in the literature, binding affinities of the six anti-apoptotic proteins towards lead anti-cancer compounds and as well to BH3-only peptides are different from each other (Sivakumar et al., 2011; Sivakumar and Sivaraman, 2012). And, in the present study, the HDNB depicted differential binding affinities and specificity towards 6 anti-apoptotic proteins whose $\mathrm{BH} 3$-binding grooves are different from each other in terms of sizes, shapes and chemical properties (as discussed above). On the other hand, an amide group and a hydroxyl group positioned at $1^{\text {st }}$ and $2^{\text {nd }}$ positions of the HDNB (Figure 1) are crucial for interacting with all the 6 anti-apoptotic proteins (though the ligand did not bind on the BH3binding groove of the $\mathrm{Bcl}-\mathrm{W}$, Table I and Figure 1). In addition, six member ring of the ligand has two nitro groups at $3^{\text {rd }}$ and $5^{\text {th }}$ positions, which were found to involve for establishing salt-bridge interactions only in Bcl-2 - HDNB and Bcl-W - HDNB complexes. In other words, the two nitro groups did not make any essential non-bonding interactions with other 4 anti-apoptotic 
proteins (Bcl-B, Bcl-XL, Bfl-1 and $\mathrm{Mcl}-1$ ). In these backgrounds, structural determinants identified for the HDNB and as well for anti-apoptotic proteins for generating protein-ligand complexes may pave ways for developing highly efficient and specific prototype molecules to each of the anti-apoptotic proteins using HDNB as seed molecule by means of 'core hopping' (Li et al., 2011) and 'de novo' (Sivakumar and Sivaraman, 2011; Rajesh and Sivaraman, 2013) drug designing strategies in near future.

\section{Conclusion}

Differential interactions of the HDNB towards the six anti-apoptotic proteins have been chiefly attributed to the differences in the chemical properties of the $\mathrm{BH}$ binding grooves of the proteins and in turn the findings may be useful on designing highly specific de novo antagonists to each of the anti-apoptotic proteins.

\section{Acknowledgements}

The authors would like to acknowledge the Centre of Excellence - AYUSH at SASTRA University, for generously allowing us to use Schrödinger software suite supported by the AYUSH research grant (Z.15015/01/2010), Government of India.

\section{References}

Bernardo PH, Wan K, Sivaraman T, Xu J, Moore FK, Hung AW, Mok HYK, Yu VC, Chai CLL. Structure-Activity Relationship Studies of phenanthridine-based Bcl-XL inhibitors. J Med Chem. 2008; 51: 6699-710.

Bernardo PH, Sivaraman T, Wan K, Xu J, Krishnamoorthy J, Sons CM, Tian L, Chin JSF, Lim DSW, Mok HYK, Yu VC, Tong JC, Chai CLL. Structural insights into the design of small molecule inhibitors that selectively antagonize mcl-1. J Med Chem. 2010; 53: 2314-18.

Bernardo PH, Sivaraman T, Wan KF, Xu J, Krishnamoorthy J, Song CM, Tian L, Chin JS, Lim DS, Mok HY, Yu VC, Tong JC, Chai CL. Synthesis of a rhodanine-based compound library targeting Bcl-XL and Mcl-1. Pure Appl Chem. 2011; 83: 723-31.

Ke N, Godzik A, Reed JC. Bcl-B, a Novel Bcl-2 family member that differentially binds and regulates Bax and Bak. J Biol Chem. 2001; 276: 12481-84.

Kelly PN, Strasser A. The Role of Bcl-2 and its pro-survival relatives in tumourigenesis and cancer therapy. Cell Death Differ. 2011; 18: 1414-24.
Labi V, Grespi F, Baumgartner F, Villunger A. Targeting the Bcl-2-regulated apoptosis pathway by $\mathrm{BH} 3$ mimetics: A breakthrough in anticancer therapy? Cell Death Differ. 2008; 15: 977-87.

Lessene G, Czabotar PE, Colman PM. Bcl-2 family antagonists for cancer therapy. Nat Rev Drug Discov. 2008; 7: 989-1000

Li XB, Wang SQ, Xu WR, Wang RL, Chou KC. Novel inhibitor design for hemagglutinin against H1N1 influenza virus by core hopping method. PLoS One. 2011; 6: e28111.

Liang H, Fesik SW. Three-dimensional structures of proteins involved in programmed cell death. J Mol Biol. 1997; 274: 291-302.

Placzek WJ, Wei J, Kitada S, Zhai D, Reed JC, Pellecchia M. A survey of the anti-apoptotic Bcl-2 subfamily expression in cancer types provides a platform to predict the efficacy of Bcl-2 antagonists in cancer therapy. Cell Death Dis. 2010; 1: e40.

Rajesh SS, Gorai B, Sivaraman T. Computational designing of novel inhibitors to Bcl-B, an anti-apoptotic protein, using fragment-based drug designing approach. Rom Biotechnol Lett. 2013; 18: 8613-21.

Rajesh SS, Sivaraman T. Cheminformatic designing of de novo inhibitors to 3-methyl adenine DNA glycosylase I (LiTagA) from Leptospira interrogans serovar lai strain 56601. Med Chem Res. 2013; 22: 3434-43.

Reed JC. Bcl-2-family proteins and hematologic malignancies: History and future prospects. Blood 2008; 111: 3322-29.

Schreiber G, Keating AE. Protein binding specificity versus promiscuity. Curr Opin Struct Biol. 2011; 21: 50-61.

Sivakumar D, Aashis R, Sivaraman, T. In silico rationalization for the differential bioavailability of ABT-737 and ABT-263 that antagonise the anti-apoptotic proteins. J Pharm Sci Res. 2011; 3: 1141-45.

Sivakumar D, Sivaraman T. In silico designing and screening of lead compounds to NS5-methyl transferase of dengue viruses. Med Chem. 2011; 7: 655-62.

Sivakumar D, Sivaraman T. Designing of de novo dual inhibitors for Bcl-XL and Mcl-1 of Bcl2-family proteins by computational methods. IEEE-International Conference on Advances in Engineering, Science and Management, ICAESM. 2012; art. no. 6216310, 174-78.

Sivakumar D, Richa T, Rajesh SS, Gorai B, Sivaraman T. In silico methods for designing antagonists to anti-apoptotic members of Bcl-2 family proteins. Mini-Rev Med Chem. 2012; 12: 1144-53.

Sivakumar D, Gorai B, Sivaraman T. Screening efficient BH3mimetics to hBcl-B by means of peptidodynmimetic method. Mol Bio Syst. 2013; 9: 700-12.

Yip KW, Reed JC. Bcl-2 family proteins and cancer. Oncogene 2008; 27: 6398-406. 